\section{CHEST November Journal club summaries}

\author{
Simon Rolin
}

\section{DIABETES AND OSA IN PREGNANCY}

In this observational case control study, polysomnography was used to objectively assess the relationship between pregnancy, obstructive sleep apnoea (OSA) and gestational diabetes mellitus (JCEM Published Online First: 21 August 2013. doi10.1210/jc.2013-2348). Comparison was made between matched groups of non pregnant nondiabetic women, pregnant women with normal glucose tolerance, and pregnant women with gestational diabetes. Pregnant women were studied during the late second trimester to early third trimester. Pregnancy alone was associated with sleep disturbance. OSA was found to be more prevalent in pregnant women with gestational diabetes than in pregnant women with normal glucose tolerance $(73 \%$ vs $27 \%, p=0.05)$. After adjustment for pre-pregnancy body mass index, the diagnosis of gestational diabetes was associated with a diagnosis of OSA (OR 6.60 (1.15-37.96)).

\section{APIXABAN FOR VTE}

Apixaban is an oral factor $\mathrm{Xa}$ inhibitor with a rapid onset of action and predictable pharmacokinetics that allow a fixeddose regimen. These characteristics may simplify the treatment of venous thromboembolism, without the need for blood test monitoring. The AMPLIFY trial ( $N$ Engl J Med 2013;369:799-808) was a randomised, double-blind study comparing apixaban with conventional therapy (subcutaneous enoxaparin, followed by warfarin) in patients with deep vein thrombosis, pulmonary embolism, or both. A fixed dose regimen of oral apixaban alone was noninferior to conventional therapy for the treatment of acute venous thromboembolism, and was associated with significantly less bleeding (RR 0.31; $95 \%$ CI 0.17 to 0.55 ).

\section{ASSOCIATION OF PSYCHOLOGICAL AND PHYSICAL SYMPTOMS IN COPD}

This study examined the association of anxiety and depression with pulmonary-

Correspondence to Simon Rolin, ST7 Respiratory and General Internal Medicine, Heart and Lung Unit, South Devon Healthcare NHS Foundation Trust, Torbay Hospital, Newton Road, Torquay, Devon TQ2 7AA, UK specific symptoms of COPD, and the extent to which disease severity and functional capacity modify this association (Int J Psychiatry Med 2013;45:189-202). Patients with COPD, enrolled in the INSPIRE-II study, were asked to complete psychosocial tests, in addition to measures of disease severity and functional capacity. After covariant analysis anxiety and depression were associated with higher levels of fatigue, shortness of breath, and frequency of COPD symptoms. The association between anxiety and shortness of breath and frequency of COPD symptoms was greater among patients with lower functional capacity.

\section{MODAFINIL FOR SLEEPINESS IN OSA}

In this Japanese double blind, placebo controlled study, patients with OSA and residual excessive sleepiness on optimal nasal CPAP, were randomised to either modafinil or placebo once daily for 4 weeks $(J$ Clin Sleep Med 2013;9:751-7). At completion of the study mean changes in ESS, and sleep latency on maintenance of wakefulness test, were significantly greater with modafinil than with placebo. ESS total score decreased from $>11$ to $<11$ in $69.2 \%$ of modafinil treated patients and $30.6 \%$ of the placebo treated group. There was no significant difference in nocturnal polysomnography between the 2 groups. Treatment with modafinil was well tolerated.

\section{PREDICTORS OF MORTALITY IN}

\section{SYSTEMIC SCLEROSIS ASSOCIATED PAH}

Pulmonary Hypertension (PAH) Assessment and Recognition of Outcomes in Scleroderma (PHAROS) is a prospective registry of systemic sclerosis (SSc) patients at high risk for $\mathrm{PAH}$, or with definite $\mathrm{PAH}$ diagnosed by right heart catheterisation, detected by routine screening for PAH at US SSc centres. This study aimed to assess cumulative survival rates, and used multivariant analysis to identify independent predictors of mortally in patients with incident SSc-PAH (Arthritis Care Res (Hoboken) Published Online First: 27 Aug 2013. doi:10.1002/acr.22121). Only patients with WHO group 1 PAH without significant interstitial lung disease were included in the analysis. 1, 2, and 3 year cumulative survival was $93 \%, 88 \%$ and $75 \%$. Age $>60$, male gender, functional class IV and DLCO <39\% predicted were significant predictors of mortality. This is the largest study describing survival in incident SSc-PAH patients followed at multiple US SSc centres. Survival rates were better than those reported in other SSc-PAH cohorts.

\section{qPCR TO DIFFERENTIATE BETWEEN PCP AND Pj}

Conventional PCR in respiratory samples does not differentiate between Pneumocystis pneumonia (PCP) and Pneumocystis jirovecii $(\mathrm{Pj})$ colonisation. In this study $\mathrm{Pj}$ real-time quantitative PCR (qPCR) was used to discriminate PCP from Pj colonisation in immunocompromised patients presenting with pneumonia (Eur J Clin Microbiol Infect Dis Published Online First: 30 Aug 2013. doi:10.1007/s10096-0131960-3). Diagnoses were retrospectively determined by a multidisciplinary group of experts blinded to the Pj qPCR results. The positive predictive value was $100 \%$ for results with more than 31600 copies $/ \mathrm{mL}$ and the negative predictive value was $100 \%$ for results with fewer than 3160 copies $/ \mathrm{mL}$. qPCR can be helpful to discriminate PCP from Pj colonisation.

\section{PREDICTORS OF TREATMENT FAILURE IN SEVERE ASTHMA}

The authors of this study performed a planned secondary analysis of data from a large multicentre trial (the $3 \mathrm{Mg}$ trial) to identify which elements of clinical assessment predicted unsuccessful treatment of severe acute asthma (Emerg Med J Published Online First: 29 Aug 2013. doi:10.1136/emermed-2013-203046).

Unsuccessful treatment was defined as the need for critical care within 7 days of attendance, or unplanned additional treatment. The strongest predictors of unsuccessful treatment in patients with severe acute asthma were PEFR (at baseline and $2 \mathrm{~h}$ after initiation of treatment), heart rate (at baseline and $2 \mathrm{~h}$ after initiation of treatment) and other serious illnesses. However the predictive ability of models based on these variables was limited.

To cite Rolin S. Thorax 2013;68:1084.

Thorax 2013;68:1084.

doi:10.1136/thoraxjnl-2013-204487 